\title{
Analysis of Possible Nonlocal Forces in Superconducting Materials
}

\author{
Elio B. Porcelli, Victo S. Filho \\ H4D Scientific Research Laboratory, São Paulo, Brazil \\ Email: elioporcelli@h4dscientific.com
}

How to cite this paper: Porcelli, E.B. and Filho, V.S. (2018) Analysis of Possible Nonlocal Forces in Superconducting Materials. Journal of Power and Energy Engineering, 6, 85-95.

https://doi.org/10.4236/jpee.2018.61007

Received: December 26, 2017

Accepted: January 28, 2018

Published: January 31, 2018

Copyright $\odot 2018$ by authors and Scientific Research Publishing Inc. This work is licensed under the Creative Commons Attribution International License (CC BY 4.0).

http://creativecommons.org/licenses/by/4.0/

(c) (i) Open Access

\begin{abstract}
In this work, we show that anomalous forces in rotating superconductor rings seem to be nonlocal in its nature, according to same theoretical framework in our previous analysis concerning to superconducting disks and toroids. Here we discuss an experiment involving rotating and angularly accelerated superconducting rings and show that the concept of generalized quantum entanglement can explain the anomaly accordingly. In fact, the hypothesis of momentum variation exchanged between Cooper pairs and outer particles regarding a hypothesis of preexisting state of generalized quantum entanglement which is also valid in this system because classical macroscopic quantities are performed in the calculation and indicate good agreement between experimental and theoretical results. We also analyze the possible reason for the discrepance between positive and null results in case of some high voltage discharge experiments involving superconducting discs in terms of nonlocal force induction aiming to reinforce that the anomalous effect can really exist in all of those superconducting systems. The experiments indicate that the anomalous forces are still weak, but our study can provide some possible physical conditions in order to increase the magnitude of the forces and provide future viable technological applications from that phenomenon.
\end{abstract}

\section{Keywords}

Cooper Pairs, Anomalous Forces, Superconductivity, Generalized Quantum Entanglement

\section{Introduction}

It is well known from literature [1] that interesting phenomena occur from the operation of superconductors of type I (in which there is one critical field) or type II (in which one can realize two critical fields). In the case of conventional 
superconductors, a well-successful theory known as Bardeen, Cooper and Schrieffer theory or BCS theory [2] explains as the electric current can move through the wires without heating or losses of energy. Such a theory predicts that the electrons in the superconducting materials move in pairs (known as pairs of Cooper) and without collisions with the atoms of the material (zero electrical resistance), being the origin of the superconductivity. In case of hightemperature materials (as YBCO ceramics), the mechanism of superconductivity seems to be more complex, but there are many evidences that Cooper pairs can also be the mechanism for the explanation of the phenomenon in many cases. Besides, the interesting phenomena as Meissner effect in type-I superconductors can also be explained in the context of the electromagnetic traditional theories of superconductors as Ginzburg-Landau and BCS theories.

However, in last years, the phenomenon of superconductivity involving superconducting ceramic materials has been investigated more profoundly in some interesting setups and indicates some novel evidences of still unexplainable phenomena, according to the traditional theories. For instance, in [3], experimental evidences of an anomaly in the local vicinity of charged high- $T_{c}$ ceramic superconductor $\mathrm{YBa}_{2} \mathrm{Cu}_{3} \mathrm{O}_{7-\mathrm{y}}$ were reported. In [4], the experimental verification of a weak gravitation shielding properties of the superconductor $\mathrm{YBa}_{2} \mathrm{Cu}_{3} \mathrm{O}_{7-\mathrm{y}}$ reinforced the existence of such anomalous forces below $70 \mathrm{~K}$ under the application of high electromagnetic fields. The experimental procedure was implemented in an improved experimental setup in order to investigate the phenomenon involving large superconductors [5]. Basically, the phenomenon is characterized by the existence of apparent anomalous forces in the vicinity of high- $T_{c}$ superconductors under non equilibrium conditions, confirmed by the improved apparatus. In other words, within the errors of the measurements, anomalous forces behave like a gravitational interaction, an effect that cannot still be explained in the framework of the known theories.

In addition, in a similar work involving anomalies from superconductors [6], it was reported other type of anomalous effect that rose from the rotation of a superconducting ring. As in the other types of forces that were generated by superconductors operating under high applied fields, the effect has not got up to date a reasonable explanation by means of the known traditional theories.

In that latter work [6], it was basically studied the anomalous effect originated from rotating superconductor rings and it raised the hypothesis that an apparent mass increase observed in the experimental measurements could be explained by the presence of an additional large gravitomagnetic field. The authors measured small acceleration fields and gravitomagnetic fields in the vicinity of a fast rotating and accelerating superconductor in order to verify their hypothesis and reported that niobium superconductors showed the first signs of such a possibility. Hence, the relevance of the phenomenon deserves a more profound experimental research as a theoretical investigation which accurately determines the magnitude of such forces and the real origin of such an anomaly. In this way, in or- 
der to better explain such experimental results with our theoretical framework, as done in the earlier reported experiments involving superconducting disks and superconducting toroids [7], here we also describe a possible explanation of this new anomalous effect involving superconducting rings in rotation based on the same idea that we earlier proposed [7]-[13]. In other words, in this work our main objective is to show that our theoretical model can adequately explain the experiments involving superconducting rotating rings.

In the following, we describe the experiments and our possible theoretical explanation for the anomalous effect. In general lines, the theoretical approach consists in considering valid the connection between quantum microscopic quantities and macroscopic observables, based on the theoretical framework of generalized quantum entanglement (GQE), as already proposed in recent studies considering other anomalous effects, as in the case of asymmetrical and symmetrical capacitors [8] [9] [10], magnetic cores [11], semiconductor laser diodes [12] and piezoelectric materials [13].

It is also important to observe that in a recent work [14] the existence of anomalous forces from superconducting ceramic materials was contested, originating a controversy. We analyzed the experiments presented in [14] which were performed by a same group involving high voltage discharge applied in superconducting discs. The first one presented a positive result, that is, some anomalous effects were detected during the discharge time. The second one presented a null result, that is, no anomalous effect was detected. Some possible difference between both setups was remarkable in order to explain the difference of results. We noted that only the setup with more similarity in comparison than Podkletnov's setup [5] presented the positive results. We also discuss that point in more details in next section, with the objective of showing that the effect is really possible and so the experiment involving rotation of superconducting rings is in fact compatible with the correspondent cases involving disks and toroids.

In the following, we describe in more details the experiment [6] whose anomalous effect we intend to describe. Further we discuss the possible reasons for the negative experimental results reported in the more recent experiment of the same group [14]. Next, we present our theoretical model in order to describe the anomalous effect in the superconducting ring experiment. At last we discuss our main conclusions.

\section{Experimental Setup}

\subsection{Experiments in Rotating and Angularly Accelerated Superconductors}

In [4], it was considered the study of rotating superconductors, in which it raised the hypothesis that beside a magnetic field proportional to its angular velocity, the so-called London moment, also a large gravitomagnetic field should appear to explain an apparent mass increase of niobium Cooper pairs. In order to investigate this so-called gravitomagnetic London moment effect, the authors meas- 
ured small acceleration fields and gravito-magnetic fields in the vicinity of a fast rotating and accelerating superconductor in their experimental facility, designed and built for this objective.

In Figure 1, we can see the details of the experimental setup. The authors reported that niobium superconductors showed the first signs within the margin of error that are consistent with the existence of gravitomagnetic London moment. The theoretical explanation proposed by them was compatible with the experimental measurements within a factor of 2. So, in order to better explain the experimental results with our theoretical framework as well, here we also describe a possible explanation of this new anomalous effect.

In Table 1, we can see the main physical properties of the superconducting ring used in the experiments. As seen in Table 1 the ring was in temperatures (4 $\mathrm{K}-6 \mathrm{~K}$ ) below the critical temperature $T_{c}$ of niobium, so that the superconducting phase was achieved under helium refrigeration. In those conditions, in one configuration of the experiment (single sensor configuration), accelerometers were placed in the interior of the ring in an arbitrary distance from its

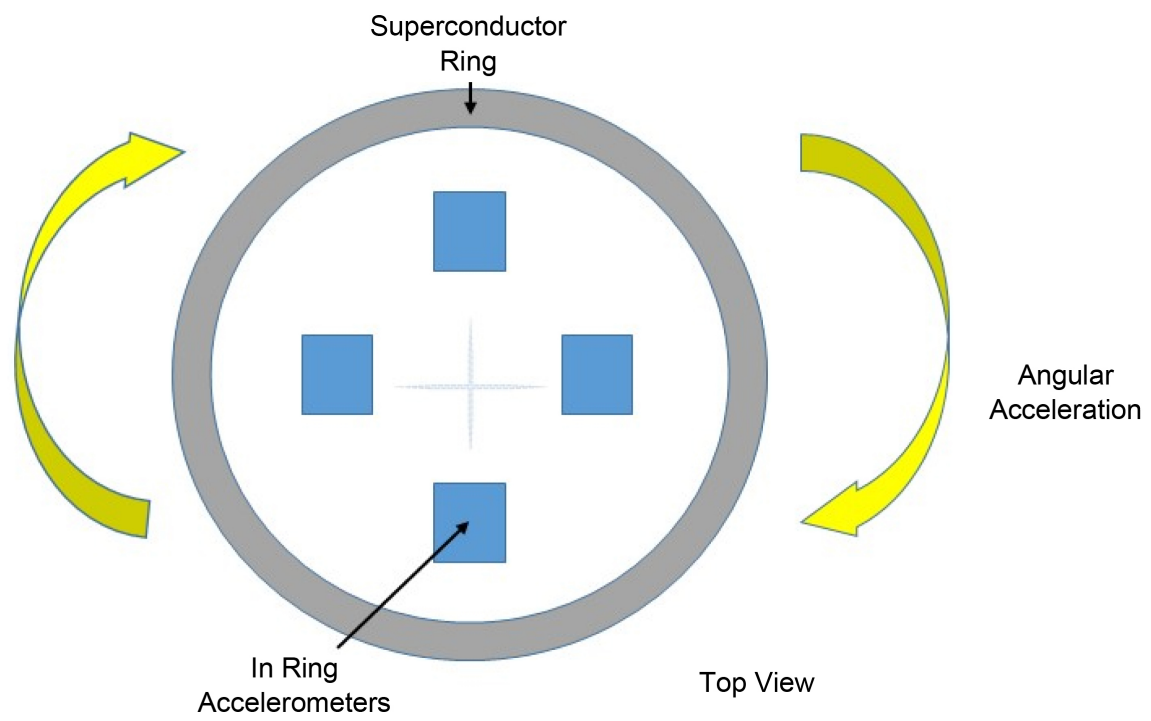

Figure 1. A simple sketch of the experimental apparatus used in [6]. The superconducting ring operates under rotation and in such conditions one experimentally detected the existence of a field of forces which resembles a weak gravitational field in its surroundings. In that paper [6], the superconducting niobium ring had the physical quantities data given in Table 1.

Table 1. Physical quantities of the superconducting niobium ring used in the experiments reported in literature [6].

\begin{tabular}{cc}
\hline External Diameter & $150 \mathrm{~mm}$ \\
Thickness & $6 \mathrm{~mm}$ \\
Height & $15 \mathrm{~mm}$ \\
Angular Acceleration & $2000 \mathrm{rad} / \mathrm{s}^{2}$ \\
Temperature & $4 \mathrm{~K}-6 \mathrm{~K}$ \\
\hline
\end{tabular}


symmetry axis $(36 \mathrm{~mm})$ and it was measured peaks of induced acceleration by the ring with magnitude proportional to its acceleration. It was not detected any signal of induced acceleration by the accelerometers with the temperature above the critical one, indicating a crucial influence of Cooper pairs in the process. From this premise, it is possible to determine the magnitude of the induced acceleration.

\subsection{Discrepancy on the Experiments Involving High Voltage Discharge Applied in Superconducting Discs}

It is relevant to report that in [14] the authors of the same group recently published a paper in which a similar experiment involving superconducting devices was implemented in an improved version of the experimental apparatus. However, they reported negative results, that is, the effect measured was null, in discrepance with their own earlier apparatus and measurements. Here we describe a trial of explaining such a discrepance.

In the first experiment of Tajmar group [6], the experimental results were positive for the presence of anomalous forces and observing the experimental setup one observes in the scheme shown in Figure 2 that it was less complex than the new one and also closer to the Podkletnov one.

However, in the second updated experimental setup the group reported negative results, as one can verify in the scheme shown in Figure 3, discussed and also presented in [14].

We can try explaining the reasons for such a different experimental measurement. The main motivation is the belief in the real existence of the anomalous forces always we have high- $T_{c}$ superconductors in many shapes and conditions of operation below the critical temperature.

In the second experiment, as one can observe in the scheme shown in Figure 3 , the authors really shield the sensor of the impulse, but one can verify two

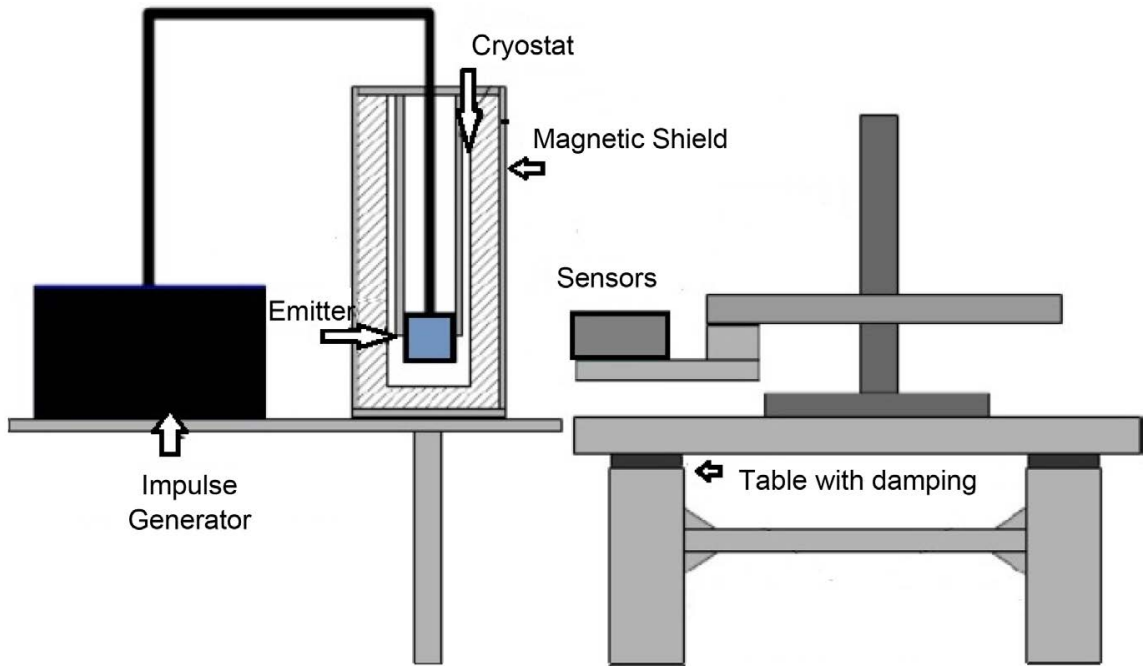

Figure 2. Scheme of the first experimental setup, adapted from [14]. The setup was built in order to measure anomalous forces in the superconducting disk. 


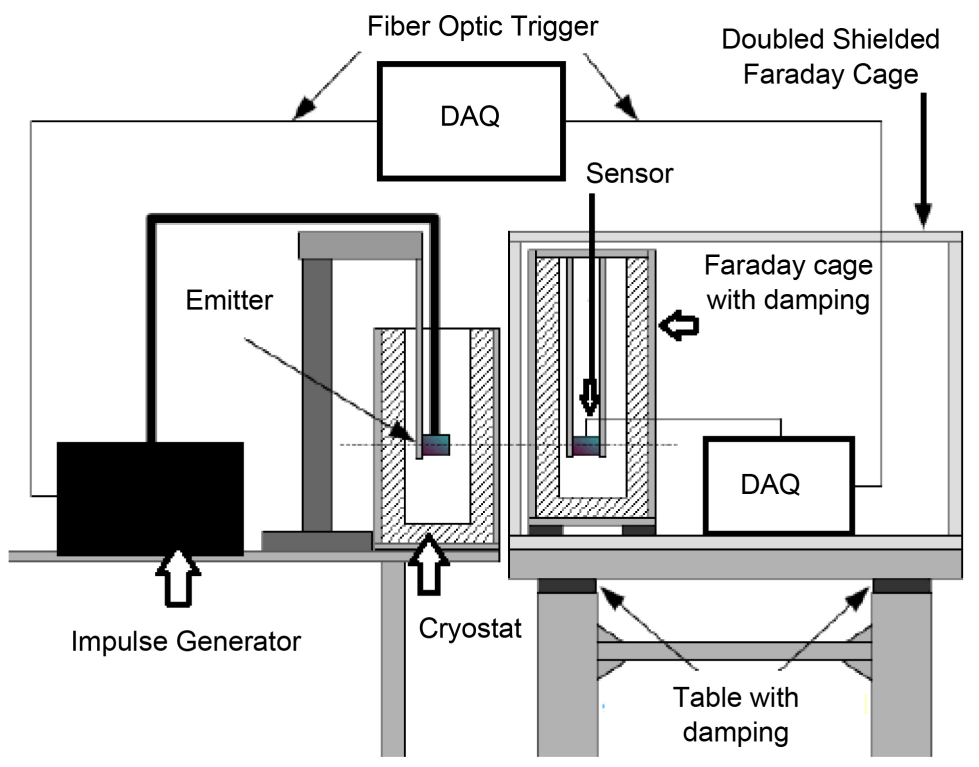

Figure 3. Scheme of the improved experimental setup, adapted from [14]. The setup was improved in order to verify the existence of anomalous force.

possible problems:

1) The emitter and the sensor are connected to that circuit by optical fiber cabs. Although they are not metallic conductors, it can occur current leakings in the SC emitter and the magnitude of the force is essential for the induction of such forces. In other words, the DAQ circuit with connection via optical fiber became a fork of current that can have significantly decreased the magnitude of the current pulse in the SC emitter.

2) The mechanical support used for the SC emitter can also have leaked current, although it was made of insulant material. In fact, the magnitude of the electric current is of order of thousands of Ampères and it is connected to the ground, hence some lesser order but significant magnitude of current can have been lost in the apparatus.

Note that the first experiment of Tajmar [6] and the experiments of Podkletnov did not present such two problems described above. So, we believe that possible current leakings can have been the cause of the problem, considering that the magnitude of the anomalous forces are very weak and hence even small losses of current can affect the experimental results.

In the next section, we describe the model proposed in order to explain the anomalous effect reported in literature and described in this section [6].

\section{Theoretical Framework}

\subsection{Explaining the Anomalous Effects in Rotating and Angularly Accelerated Superconducting Rings}

Our hypothesis in the description of the effect concerns to momentum exchange between Cooper pairs and the outer environment. Here our model is based on the existence of Cooper pairs in high- $T_{c}$ superconductors, that is, we suppose 
that the effect is really caused by coupling of electrons in pairs as occurs in metallic superconductors. Such a hypothesis is based on some works in the literature [15], it was reported that many materials are not well described by the BCS model, but the pairing theory is a wider concept in which fermions can be described by means of an effective attractive interaction that produces condensates with large overlaps between pairs. Hence, the BCS concept is not excluded as a possible explanation of the behavior of non-metallic superconductors.

In other work [16], it was studied the mechanism responsible for superconductivity in high-temperature cuprates and reported that evidences indicated that the pairing state could exist but in a new form, characterized by an anisotropic order parameter. Besides it was reported that a class of experiments involving the most widely studied cuprate $\left(\mathrm{YBa}_{2} \mathrm{Cu}_{3} \mathrm{O}_{7-\mathrm{x}}\right)$ and based on the interference of the quantum-mechanical phases in Josephson tunnel junctions and $\mathrm{dc}$ SQUID devices gave strong evidence for pairing in a channel with d-wave symmetry. Further other recent work [17] also suggests the presence of the mechanism of Cooper-pairing in layered high- $T_{c}$ superconductors. In other words, by considering all the works that reinforce the presence of electron pairing in high$T_{c}$ superconductors, we really adopt such a hypothesis in our model and indistinctly call them Cooper pairs from now on.

If we consider the dimensions of the superconducting ring and the niobium density $\left(8.57 \mathrm{~g} / \mathrm{cm}^{3}\right)$ it is possible to calculate its mass $(178.098 \mathrm{~g})$ and the inertia momentum by

$$
I=m r^{2}
$$

in which $I$ is the inertia momentum of the ring and $m$ is the mass and $r$ is its radius $\left(7.35 \times 10^{-2} \mathrm{~mm}\right)$. From this equation, we obtained $I=0.00096213 \mathrm{~kg} \cdot \mathrm{m}^{2}$. The value of the torque raised from the angular acceleration of the ring can be calculated from

$$
T=I \frac{\mathrm{d} w}{\mathrm{~d} t}
$$

and the tangential force related to the torque $T$ can be obtained from

$$
F=\frac{T}{r}
$$

So, we obtained the values $T=0.9621 \mathrm{~N} \mathrm{~m}$ and $F=13.09 \mathrm{~N}$. We can extract the information of the relationship between the mass of Cooper pairs and the total mass of the superconducting ring by means of the relationship between the densities:

$$
\frac{d^{*}}{d}=1.95 \times 10^{-6}
$$

in which $d^{*}$ is the density of Cooper pairs and $\mathrm{d}$ is the total density. The tangential force $F^{*}$ specifically inerent to Cooper pairs can be determined by

$$
F^{*}=\frac{F d^{*}}{d}
$$


The value found is $F^{*}=2.552589 \times 10^{-5} \mathrm{~N}$. Finally, the induced acceleration by the superconducting ring in the four accelerometers with $6.7 \mathrm{~g}$ each can be calculated by

$$
a=\frac{F^{*}}{m}
$$

in which $m$ is the total mass of the four accelerometers $(26.8 \mathrm{~g})$, so that $a=0.953 \times$ $10^{-3} \mathrm{~m} / \mathrm{s}^{2}$. The model of accelerometers used in the experiment due to their accuracy has their technical specifications indicated in a technical report [18]. The theoretical value of the induced acceleration-which is the tangential acceleration of internal Cooper pairs in the superconducting ring-presented a value $a_{\text {ind }}=$ $0.953 \times 10^{-3} \mathrm{~m} / \mathrm{s}^{2}$. Such a magnitude is in good agreement with the experimental one $\left(0.98 \times 10^{-3} \mathrm{~m} / \mathrm{s}^{2}\right)$, as indicated in figure 6 of a recent work of Tajmar [6]. For an angular acceleration $w=500 \mathrm{rad} / \mathrm{s}^{2}$ one has an experimental value $a_{\text {ind }}=$ $0.4905 \times 10^{-3} \mathrm{~m} / \mathrm{s}^{2}$ and the calculation of the theoretical value provides the magnitude $a_{\text {ind }}=0.476 \times 10^{-3} \mathrm{~m} / \mathrm{s}^{2}$. Such magnitudes demonstrate the good agreement of the simple calculations without a consideration of geometric factors. If this line of thinking is right, the preexisting quantum entanglements among Cooper pairs under angular acceleration (tangential) formed in the interior of the superconducting ring below the critical temperature $T_{c}$ induce in the internal accelerometers peaks of acceleration, even that they are weak. It is worth to report that in the experiments there was careful attention in the implementation so that it was excluded the possibility of trivial interferences. Besides, in relation to the theoretical framework early indicated in the literature, the difficulty of the explanation of the anomalous effects by means of gravito-electric fields hypothesis shows that our theoretical proposal represents at least a possible complement or even a main line of research in the investigation of the physical mechanisms that generates the phenomenon.

\subsection{Explaining the Anomalous Effects in Direct Current Discharges}

It is not possible to accurately calculate the magnitude of the supposed nonlocal forces induced in the accelerometers by means of the available data from the articles in the literature [14]. So, it is not viable to compare the theoretical predictions with the experimental measurements of the accelerations.

But a peculiar anomalous effect that occurred in the measurements was the destruction of the superconducting disks during the application of current peaks whose values were very high.

In [19], we found values around $12 \mathrm{~N} / \mathrm{mm}^{2}$ for the tensile strength of the ceramics used in the experiment and operating in their superconducting state.

Our theoretical model predicts that the nonlocal force which induces charge carriers in conductors or superconductors can be the responsible for those effects if elevated values of electric current occur, as already investigated by Peter Graneau [20] in the case of wires breaking phenomenon. The magnitude of the 
nonlocal force can be calculated by using classical quantities, as discussed in our previous work involving superconductors [7]:

$$
F=\mu_{0} I^{2}
$$

in which $F$ is the magnitude of the nonlocal force (in $\mathrm{N}$ ) inducing charge carriers and the proportionality constant represents the magnetic permittivity of the vacuum and $I$ is the magnitude of the electric current in the conductor.

Three setups were built using a YBCO ceramic disk of $22 \mathrm{~mm}$ diameter in its superconducting phase where were applied direct current discharges with peak values between $4000 \mathrm{~A}$ e $7000 \mathrm{~A}$. Those values correspond to nonlocal inductor forces between $20.10 \mathrm{~N}$ and $61.57 \mathrm{~N}$. So, this leads us to values of tensile strength between $5.81 \mathrm{~N} / \mathrm{mm}^{2}$ a $16.2 \mathrm{~N} / \mathrm{mm}^{2}$, which can clearly overcome the limits of resistence of the YBCO ceramic, as earlier indicated, and then explain its destruction.

The fact of non-occurrence of the destruction of the YBCO superconducting disk in the first setup can be explained by our theoretical model if we take into account that the device presents a very lower value of current (200 A) magnetically induced via solenoid. Hence the correspond induced anomalous force has also a much lower value, so that the magnitude of the tensile strength gets much lower than the limit of the superconducting ceramic used in the experiments.

\section{Conclusions and Final Remarks}

In this work, we present a new model in order to theoretically explain in qualitative and quantitative terms some experiments concerning to anomalous forces externally induced by superconductors built in shape of rings and disks operating in different conditions below the critical temperature. Our main objective in the present paper is the proposal of a model based on the same concept described in early similar works, that is, the microscopic origin of the anomalous effects in each physical system considered is due to the generalized quantum entanglements. As earlier considered, we can use classical analysis in the calculation of the physical quantities and show that the empirical formulas for the anomalous forces generated by forces generated by the superconductor operation in case of angularly accelerated rings or in case of discs over high voltage discharge can predict very good results, in accordance with the experimental range measured for those experiments. Our model considers a preexisting state of generalized quantum entanglement so that the Cooper pairs formed below the critical temperature of superconductors can exchange collectively their momentum variation with outer particles composing macroscopic objects such as the mass test of gyroscopes or accelerometers.

The results obtained from our theoretical proposal indicate a good agreement with the experimental values presented in [6]. Such a good accuracy of our calculations for the magnitude of the induced nonlocal forces reinforces the concept of the application of classical quantities related to the macroscopic observables in the framework of the generalized quantum entanglement (GQE), as 
similarly described in other previous works in which other anomalous effects were analyzed.

\section{References}

[1] Tinkham, M. and McKay, G. (1996) Introduction to Superconductivity. McGrawHill, Inc., Pennsylvania Plaza, 1-16. https://doi.org/10.1063/1.2807811

[2] Bardeen, J., Cooper, L. and Schriffer, J.R. (1957) Theory of Superconductivity. Physical Review, 108, 1175. https://doi.org/10.1103/PhysRev.108.1175

[3] Podkletnov, E. and Modanese, G. (2001) Impulse Gravity Generator Based on Charged $\mathrm{YBa}_{2} \mathrm{Cu}_{3} \mathrm{O}_{7-\mathrm{y}}$ Superconductor with Composite Crystal Structure. https://arxiv.org/abs/physics/0108005

[4] Podkletnov, E. (1997) Weak Gravitation Shielding Properties of Composite Bulk $\mathrm{YBa}_{2} \mathrm{Cu}_{3} \mathrm{O}_{7-\mathrm{y}}$ Superconductor Below $70 \mathrm{~K}$ under e.m. Field. https://arxiv.org/abs/cond-mat/9701074

[5] Podkletnov, E. and Modanese, G. (2003) Investigation of High Voltage Discharges in Low Pressure Gases through Large Ceramic Superconducting Electrodes. Journal of Low Temperature Physics, 132, 239-259. https://doi.org/10.1023/A:1024413718251

[6] Tajmar, M., Plesescu, F., Seifert, B. and Marhold, K. (2007) Measurement of Gravitomagnetic and Acceleration Fields around Rotating Superconductors. AIP Conference Proceedings, 880, 1071. https://doi.org/10.1063/1.2437552

[7] Porcelli, E.B. and Filho, V.S. (2017) Theoretical Study of Anomalous Forces Externally Induced by Superconductors. Natural Science, 9, 293-305.

https://doi.org/10.4236/ns.2017.99028

[8] Porcelli, E.B. and Filho, V.S. (2016) On the Anomalous Forces in High-Voltage Symmetrical Capacitors. Physics Essays, 29, 2-9. https://doi.org/10.4006/0836-1398-29.1.002

[9] Porcelli, E.B. and Filho, V.S. (2015) On the Anomalous Weight Losses in HighVoltage Symmetrical Capacitors. https://arxiv.org/abs/1502.06915

[10] Porcelli, E.B. and Filho, V.S. (2016) Characterization of Anomalous Asymmetric High-Voltage Capacitors. IET Science, Measurement \& Technology, 10, 383-388. https://doi.org/10.1049/iet-smt.2015.0250

[11] Porcelli, E.B. and Filho, V.S. (2016) Anomalous Effects from Dipole-Environment Quantum Entanglement. International Journal of Advanced Engineering Research and Science (IJAERS), 4, 131-144. https://doi.org/10.22161/ijaers.4.1.21

[12] Porcelli, E.B. and Filho, V.S. (2017) Induction of Forces at Distance Performed by Semi-Conductor Laser Diodes. American Journal of Engineering Research (AJER), 6, 35-48.

[13] Porcelli, E.B. and Filho, V.S. (2016) Induction of Force Performed by Piezoelectric Materials. https://arxiv.org/abs/1612.04201

[14] Lörincz, I. and Tajmar, M. (2015) Design and First Measurements of a Superconducting Gravity-Impulse-Generator. 51 st AIAA/SAE/ ASEE Joint Propulsion Conference, AIAA Propulsion and Energy Forum, AIAA 2015-4080.

Lörincz, I. and Tajmar, M. (2016) Null Results of a Superconducting Gravity-Impulse Generator. 52nd AIAA/SAE/ ASEE Joint Propulsion Conference, July 25-27 2016, Salt Lake City, UT. https://doi.org/10.2514/6.2016-4988

[15] Dagotto, E. (1994) Correlated Electrons in High-Temperature Superconductors. Reviews of Modern Physics, 66, 763-840. 
https://doi.org/10.1103/RevModPhys.66.763

[16] Van Harlingen, D.J. (1995) Phase-Sensitive Tests of the Symmetry of the Pairing State in the High-Temperature Superconductors-Evidences for Symmetry $\mathrm{d}_{\mathrm{x}-\mathrm{y}}^{2}$. Reviews of Modern Physics, 67, 515-535. https://doi.org/10.1103/RevModPhys.67.515

[17] Tavkhelidze, A.A. (2011) Mechanism of Cooper-Pairing in Layered High Temperature Superconductors. http://lanl.arxiv.org/abs/1109.3978

[18] Colibrys (2016) Smart Sensing Solutions Datasheet. http://www.colibrys.com

[19] Krabbes, G., Fuchs, G., Canders, W.-R., May, H. and Palka, R. (2016) High Temperature Superconductor Bulk Materials: Fundamentals, Processing, Properties Control, Application Aspects. John Wiley \& Sons, Hoboken.

[20] Bueno, M. and Assis, A.K.T. (2015) Cálculo de Indutância e de Força em Circuitos Elétricos [Calculation of Inductance and Force between Electric Circuits]. 2nd Edition, Roy Keys Inc., Apeiron Montreal, Canada. 